\title{
A comparative study on patient specific absolute dosimetry using slab phantom, acrylic body phantom and goat head phantom
}

\author{
Om Prakash Gurjar, Surendra Prasad Mishra \\ Department of Physics, Mewar University, Chittorgarh, India
}

Received January 06, 2015; Revised February 17, 2015; Accepted February 28, 2015; Published Online April 04, 2015

\section{Original Article}

\begin{abstract}
Purpose: To compare the results of patient specific absolute dosimetry using slab phantom, acrylic body phantom and goat head phantom. Methods: Fifteen intensity modulated radiotherapy (IMRT) plans already planned on treatment planning system (TPS) for head-and-neck cancer patients were exported on all three kinds of phantoms viz. slab phantom, acrylic body phantom and goat head phantom, and dose was calculated using anisotropic analytic algorithm (AAA). All the gantry angles were set to zero in case of slab phantom while set to as it is in actual plan in case of other two phantoms. All the plans were delivered by linear accelerator (LA) and dose for each plan was measured by $0.13 \mathrm{cc}$ ion chamber. The percentage (\%) variations between planned and measured doses were calculated and analyzed. Results: The mean \% variations between planned and measured doses of all IMRT quality assurance (QA) plans were as 0.65 (Standard deviation (SD): 0.38 ) with confidence limit (CL) 1.39, 1.16 (SD: 0.61 ) with CL 2.36 and 2.40 (SD: 0.86) with CL 4.09 for slab phantom, acrylic head phantom and goat head phantom respectively. Conclusion: Higher dose variations found in case of real tissue phantom compare to results in case of slab and acrylic body phantoms. The algorithm AAA does not calculate doses in heterogeneous medium as accurate as it calculates in homogeneous medium. Therefore the patient specific absolute dosimetry should be done using heterogeneous phantom mimicking density wise as well as design wise to the actual human body.
\end{abstract}

Keywords: Acrylic Body Phantom; Goat Head Phantom; Patient Specific Absolute Dosimetry

\section{Introduction}

In the era of modern radiotherapy techniques day to day new and advance techniques of radiotherapy are being implemented like intensity modulated radiotherapy (IMRT), image guided radiotherapy (IGRT), rapid arc therapy and now flattening filter free (FFF). In every technique advance features and benefits for patients are claimed. One of the most important parts of treatment planning is algorithm which calculates the dose using information from computed tomography (CT) images and structures contoured. Different algorithms have already been compared on the basis of their accuracy in dose calculation without/with density correction and the clinical impact of these algorithms. ${ }^{1}$ Anisotropic analytical algorithm (AAA) is considered more accurate compared to pencil beam convolution algorithm as it has compatibility of doing better heterogeneous correction. ${ }^{2}$

AAA is based on the superposition-convolution method which is widely being used in the ECLIPSE (Varian Medical Systems, Palo Alto, CA) TPS. The AAA employs spatially-variant MC derived convolution scatter kernels, and it has separate modeling for primary photons, scattered extra focal photons and contaminant electrons. With the help of Radio- logical scaling of the dose deposition function in the beamlet direction inhomogeneities in the medium are accounted for anisotropically in the 3 dimensional vicinity and electron density based scaling of the photon scatter kernels in 16 lateral directions. With the superposition of doses of the photon and electron convolutions the resultant dose distribution is achieved. ${ }^{3,}{ }^{4}$ However still researchers are doing work on many codes of Monte Carlo, they advocate that Monte Carlo consider more accurately the higher level of scattering and effect of heterogeneity. ${ }^{5}$ Recently the new algorithm Acuros $\mathrm{XB}$ (AXB) has been introduced with ECLIPSE TPS which is considered similar to Monte Carlo, few authors have already presented AXB more accurate algorithm compare to AAA.6,7 The research in this direction of developing new radiotherapy modalities with more accurate dose calculating algorithms is going on.

Before patient treatment with the plan done on TPS, patient specific relative and absolute dosimetry is done. There are so many methods of doing relative dosimetry e.g. film dosimetry by using commercially available film quality assurance (QA) dosimetry system OmniPro-I'MRT, dosimetry using elec- 
tronic portal imaging device (EPID) dosimetry etc. ${ }^{8-11}$ For absolute dosimetry most of people are using small volume ion chamber and homogeneous phantoms. ${ }^{12,} 13$ The plan already done for patient on TPS is exported on phantom CT-images and dose is re-calculated without changing the monitor units (MU). Since the medium of phantom is of homogeneous density so same kind of interaction of radiation inside the phantom volume happens and so algorithm in use calculate dose more accurately and when plan is delivered on linear accelerator (LA) and dose measured with planned dose, the variation between planned and measured dose lives very low (in most of cases $<1 \%$ ). But medium of the actual patient body is heterogeneous having bones, soft tissue, air cavity, etc. Hence, calculated dose by algorithm in so complicated volume must be verified by similar kind of heterogeneous body phantom. In this study patient specific absolute dosimetry has been done by using three kinds of phantoms viz. slab phantom, acrylic body phantom, and goat head.

\section{Methods and Materials}

Three kinds of phantoms were chosen for the patient specific absolute dosimetry of IMRT plans already done for the treatment. First one was slab phantom "solid phantom SP34" (IBA Dosimetry $\mathrm{BmbH}$, Schwarzenbruck, Germany) as shown in Figure 1(A), each slab of which was made of polystyrene $\mathrm{C} 8 \mathrm{H} 8$ with effective atomic number 5.74. The second phantom was acrylic head phantom (TOPSLANE International, USA) as shown in Figure 1 (B), the mean Hounsfield number (HU) measured from TPS was 112 (density 1.112 $\mathrm{gm} / \mathrm{cc}$ ) and third phantom used was the goat head (thrown by meat shops as waste material) as shown in Figure 1 (C). This goat head phantom had all the internal structures as it is i.e. bones, tissues and air cavity. The density of the internal organs of this goat head was equivalent to that of human head. ${ }^{14}$

This phantom was wrapped in polythene and thermoplastic sheet (Orfit) was molded in required shape to hold it on base plate in stationary position. Ion chamber was fixed in cavity prepared by screw driver at approximately geometrical centre of phantom volume and was kept at the same position till the end of experiment. Three fiducial lead markers were put on two bilateral points and one anterior point on surface of phantom in same cross-sectional plan to make three reference points. The goat head phantom was preserved in refrigerator during the period of creating plan on CT images of phantom. The patient specific absolute dosimetry using these three phantoms was done on three different days but all the selected reference plans (15 IMRT plans) were same for all three phantoms.

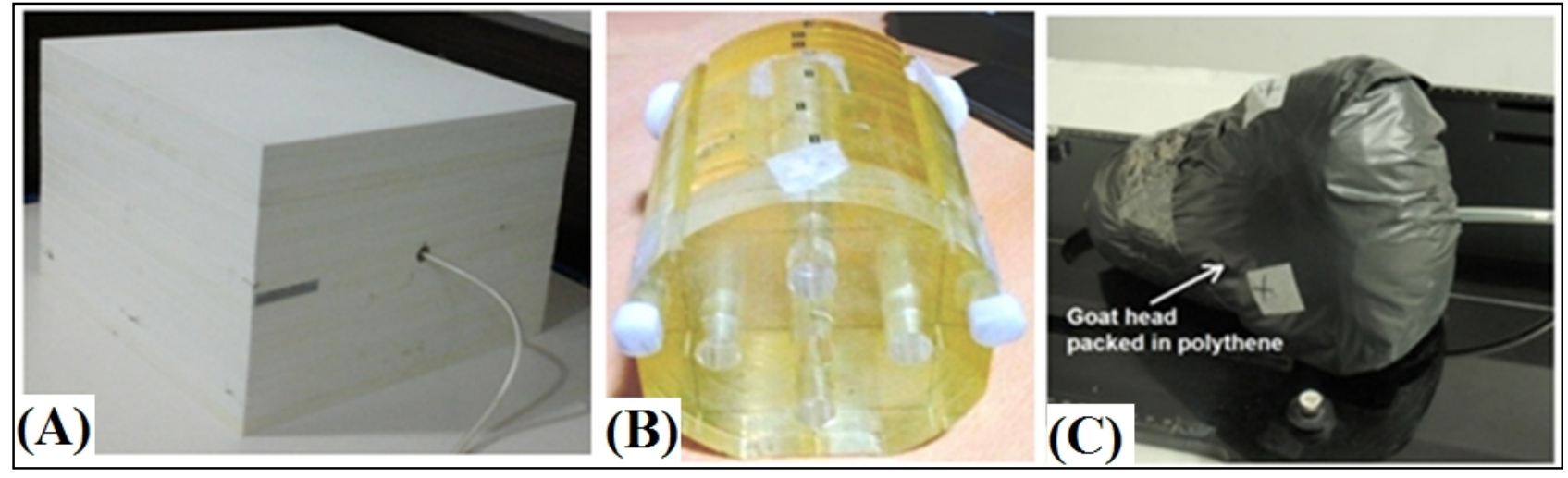

FIG. 1: Photographs of (A) Slab phantom, (B) Acrylic body phantom and (C) Goat head phantom.
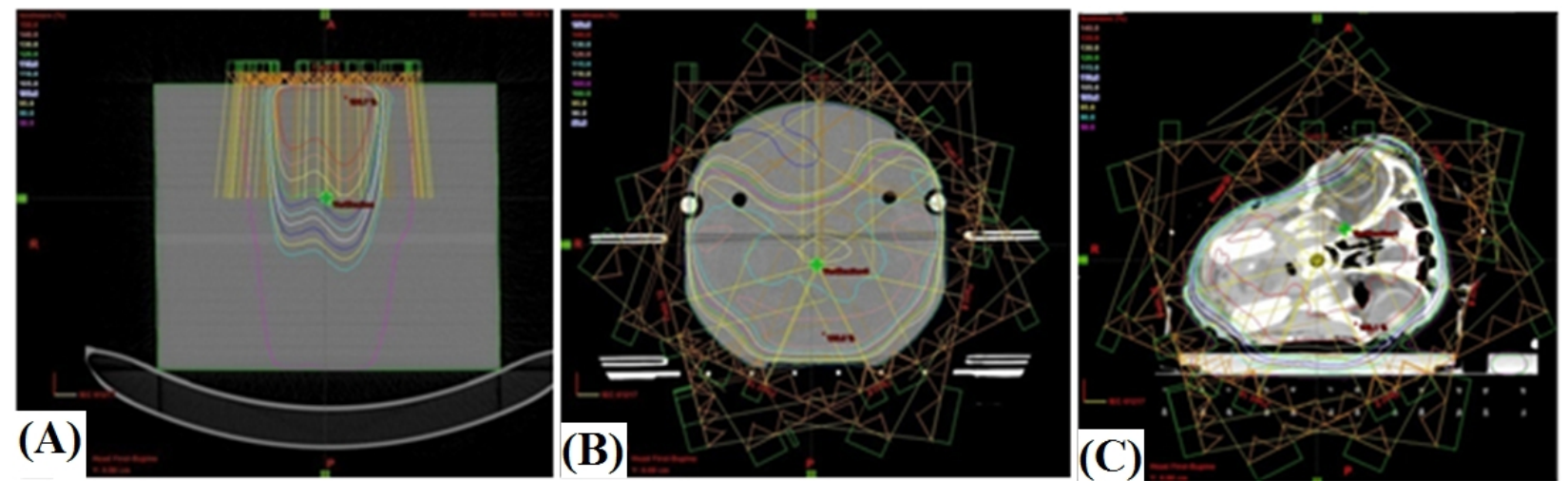

FIG. 2: Computed tomography slices of (A) Slab phantom, (B) Acrylic body phantom and (C) Goat head phantom showing dose distribution. 
The CT of phantoms was done on Siemens SOMATOM Definition AS scanner (Siemens Medical System, Germany) with slice thickness of $3 \mathrm{~mm}$ for the planning purpose. The CT images were imported on TPS system ECLIPSE Vs. 8.9 (Varian Medical Systems, Palo Alto, CA) and fifteen IMRT plans already done for patient treatment were exported on phantoms. Since the slab phantom has plan surfaces and sharp edges and hence as a standard practice, all the gantry angles were set to zero in case of slab phantom, while set all the gantry angles as it is in actual plan in case of other two phantoms. All the selected plans were done with $6 \mathrm{MV}$ photon beam, and field arrangement was done in such a way that all fields were coplanar (CP) with couch angle $0^{\circ}$ and no parallel opposed fields were placed, 5 to 9 gantry angles were chosen from $0^{\circ}$ to $360^{\circ}$ as per requirement of plans. For larger target volumes, two fields at each gantry angle were also used because of the limitation of MLC movement in $\mathrm{x}$-direction which is $14.5 \mathrm{~cm}$, if field size exceeds than this size, it gets split into two fields automatically. ${ }^{14}$ In this way maximum of 18 fields at nine gantry angles were used in some plans. Dose volume optimizer (DVO) was used for plan optimization and anisotropic analytical algorithm (AAA) vs. 8.9.08 with grid size $0.25 \mathrm{~cm}$ was used for dose calculation. Figure $2(\mathrm{~A}-\mathrm{C})$ shows the CT slices with isodose lines of slab phantom, acrylic body phantom and goat head phantom.

After successful creation of plans and approval on RT-Chart, all the plans were scheduled on time planner for delivery. The phantoms were set on IGRT exact couch of LA with the help of reference lines on artificial phantoms and markers on goat head phantom and aligned with the help of patient-positioning system "Astor green" (LAP GmbH, Luneburg). To verify the position cone beam computed tomography (CBCT) was done using On-Board-Imaging System (OBI System, Varian Medical Systems, Palo Alto, CA) with half-bow-tie filter and compared with reference CT images. The shift in position was corrected. In the case of goat head phantom, soft tissue to soft tissue and bone to bone were matched along with ion chamber which proved that tissue deformation had not happen during the period from CT imaging to plan delivery as phantom was preserved at low temperature.

All the plans were delivered and dose for each plan was measured using DOSE1 electrometer (IBA Dosimetry, Germany) connected with $0.13 \mathrm{cc}$ ionization chamber (IBA Dosimetry, Germany), which was fixed in phantoms. The percentage (\%) variation between measured (on LA) and planned (on TPS) doses was calculated by following formula;

$\%$ variation $=($ measured dose - planned dose $) /$ planned dose $\times 100$

\section{Results}

The mean percentage variations between planned and measured doses of all IMRT QA plans were as 0.65 (Standard deviation (SD): 0.38) with confidence limit (CL) 1.39, 1.16 (SD: 0.61) with CL 2.36 and 2.40 (SD: 0.86) with CL 4.09 for slab phantom, acrylic head phantom and goat head phantom respectively. Results in details are given in the Table 1.

TABLE 1: Percentage variation between planned doses on TPS and measured doses on LA using slab phantom, acrylic head phantom and goad head phantoms.

\begin{tabular}{|c|c|c|c|c|c|c|c|c|c|}
\hline \multirow{2}{*}{$\begin{array}{l}\text { Plan } \\
\text { No. }\end{array}$} & \multicolumn{3}{|c|}{ QA plan done on Slab phantom } & \multicolumn{3}{|c|}{$\begin{array}{l}\text { QA plan done on acrylic body } \\
\text { phantom }\end{array}$} & \multicolumn{3}{|c|}{ QA plan done on goat head phantom } \\
\hline & $\begin{array}{l}\text { Planned } \\
\text { dose(cGy) }\end{array}$ & $\begin{array}{l}\text { Measured } \\
\text { dose(cGy) }\end{array}$ & $\begin{array}{l}\% \text { varia- } \\
\text { tion }\end{array}$ & $\begin{array}{l}\text { Planned } \\
\text { dose }(\mathrm{cGy})\end{array}$ & $\begin{array}{l}\text { Measured } \\
\text { dose(cGy) }\end{array}$ & $\begin{array}{l}\% \text { varia- } \\
\text { tion }\end{array}$ & $\begin{array}{l}\text { Planned } \\
\text { dose(cGy) }\end{array}$ & $\begin{array}{l}\text { Measured } \\
\text { dose(cGy) }\end{array}$ & $\begin{array}{l}\% \text { varia- } \\
\text { tion }\end{array}$ \\
\hline 1 & 234.40 & 233.88 & $0.22(-)$ & 249.80 & 252.28 & $0.99(+)$ & 259.30 & 264.30 & $1.93(+)$ \\
\hline 2 & 149.00 & 148.73 & $0.18(-)$ & 158.10 & 156.47 & $1.03(-)$ & 168.93 & 163.20 & $3.39(-)$ \\
\hline 3 & 184.60 & 185.13 & $0.29(+)$ & 204.60 & 201.07 & $1.73(-)$ & 206.50 & 202.16 & $2.1(-)$ \\
\hline 4 & 182.70 & 180.62 & $1.14(-)$ & 202.80 & 198.05 & $2.34(-)$ & 211.50 & 202.31 & $4.35(-)$ \\
\hline 5 & 189.60 & 187.94 & $0.88(-)$ & 209.90 & 206.98 & $1.39(-)$ & 227.50 & 221.38 & $2.69(-)$ \\
\hline 6 & 201.57 & 200.43 & $0.57(-)$ & 221.65 & 224.81 & $1.43(+)$ & 225.50 & 220.27 & $2.32(-)$ \\
\hline 7 & 153.90 & 154.87 & $0.63(+)$ & 187.10 & 188.47 & $0.73(+)$ & 167.60 & 171.47 & $2.31(+)$ \\
\hline 8 & 196.60 & 195.48 & $0.57(-)$ & 216.50 & 214.79 & $0.79(-)$ & 229.60 & 225.31 & $1.87(-)$ \\
\hline 9 & 178.94 & 179.15 & $0.12(+)$ & 191.30 & 192.24 & $0.49(+)$ & 203.01 & 204.93 & $0.95(+)$ \\
\hline 10 & 126.84 & 125.14 & $1.34(-)$ & 135.72 & 133.12 & $1.92(-)$ & 139.47 & 135.24 & $3.03(-)$ \\
\hline 11 & 179.84 & 177.85 & $1.11(-)$ & 192.43 & 188.93 & $1.82(-)$ & 196.60 & 192.10 & $2.29(-)$ \\
\hline 12 & 187.93 & 186.27 & $0.88(-)$ & 195.99 & 194.05 & $0.99(-)$ & 208.50 & 204.26 & $2.03(-)$ \\
\hline 13 & 183.74 & 184.92 & $0.64(+)$ & 193.06 & 193.68 & $0.32(+)$ & 201.10 & 204.91 & $1.89(+)$ \\
\hline 14 & 176.86 & 177.48 & $0.35(+)$ & 186.70 & 187.19 & $0.26(+)$ & 190.90 & 184.32 & $3.45(-)$ \\
\hline 15 & 171.54 & 173.03 & $0.87(+)$ & 180.12 & 182.17 & $1.14(+)$ & 184.60 & 187.20 & $1.41(+)$ \\
\hline \multicolumn{3}{|c|}{ Mean $\%$ variation } & 0.65 & & & 1.16 & & & 2.40 \\
\hline \multicolumn{3}{|c|}{ Standard deviation (SD) } & 0.38 & & & 0.61 & & & 0.86 \\
\hline \multicolumn{3}{|c|}{$\begin{array}{l}\text { Confidence limit } \\
(\mid \text { mean } \mid+1.96 \sigma)\end{array}$} & 1.39 & & & 2.36 & & & 4.09 \\
\hline
\end{tabular}




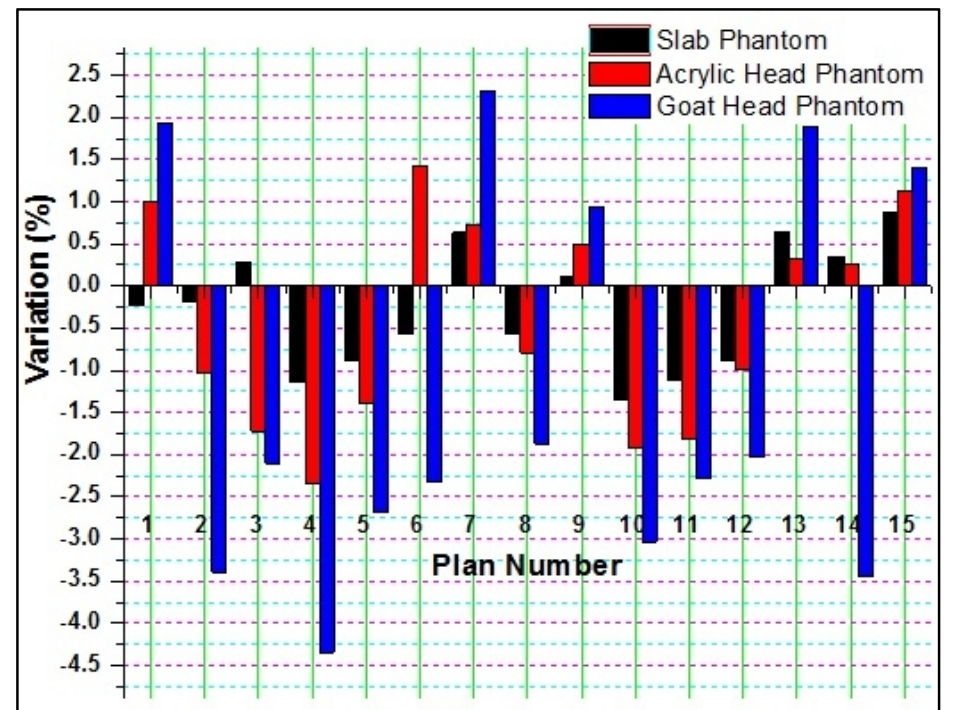

FIG. 3: Comparative graph of percentage variation between planned doses on TPS and measured doses on LA using slab phantom, acrylic head phantom and goad head phantom.

\section{Discussion}

The percentage variation between planned and measured doses in the case of slab phantom is less than $1 \%$ in most of the cases while less than two in some cases. This variation in the case of acrylic head phantom is slightly higher than in the case of slab phantom, although it is very well within the tolerance limit $( \pm 3 \%)$ prescribed in International Commission on Radiation Units and Measurements (ICRU) 83. ${ }^{15}$ The variation in the case of goat heat phantom is up to $4 \%$ in some cases which is higher than the tolerance limit. The results of slab phantom and head phantom indicates that the gantry angles in QA plan also play an important role, it is as in the case of slab phantom all the gantry angles were put at $0^{0}$ which means there is no any obstacle in the path of beam and phantom while in the case of head phantom, the gantry angles were put as these were in actual patient plans. So, in the many gantry angles base plat and other immobilizing accessories come in path of the beam. If we compare the results of these two phantoms (with homogeneous density) with the results of heterogeneous phantom goat head phantom, then we see that the large difference in the results is due to the effect of heterogeneous medium. Figure 3 represents the comparative graph of percentage variation between planned and measured doses using three kinds of phantoms.

Presently practicing most of the Physicists follows the well stabilized practices based on different guidelines like ICRU 83, TRS 398 and AAPM Task Group Report 120.15-17 The phantoms suggested in these guidelines are water equivalent because the adult human body contains $\sim 65 \%$ of water, this varies by age, sex and amount of fat in body composition. ${ }^{18}$ The newborn infant contains $73 \%$ and some obese people are as little as $45 \%$ water by weight. ${ }^{19}, 20$ So, a phantom with having its full volume of water equivalent density cannot represent human body. Also, the dose calculation in a medium having single density is a easy task for any kind of dose calculation algorithm, so when the QA plan is created on such phantoms, the algorithm calculates dose more accurately and when the plan is delivered and dose is measured in such phantoms then very less variation between measured and planned doses are obtained as seen in this study.

The human body is comprises of different density mediums e.g. fat, bones, air cavities and tissue. ${ }^{21}$ Due the variation in electron density of two mediums, the dose deposition at the interface of two mediums varies by significant value. Since the density of bone is high compare to soft tissue so the production of secondary electron in bones is also higher and so the dose at bone - soft tissue interface is higher and similar kind of phenomena happens at the interface of all two mediums with different densities. ${ }^{22,}{ }^{23}$ One of the biggest task for dose calculation algorithms is heterogeneity. Currently available TPS have advanced and more accurate algorithms which apply the heterogeneity correction factor while doing dose calculation like AAA. To verify the accuracy of dose calculated by these algorithms in case of each patient, the patient specific absolute dosimetry should be done by using heterogeneous phantom mimicking density of human body.

\section{Conclusion}

The variation in planned and measured doses is extremely higher in goat head phantom compare to the variation in the case of slab and acrylic head phantom. The variation is little higher in acrylic head phantom compare to the slab phantom. This study concludes that the dose calculating algorithms AAA does not calculate doses in heterogeneous medium as accurate as it calculates in homogeneous medium. Therefore the patient specific absolute dosimetry should be done using 
heterogeneous phantom mimicking density wise as well as design wise to the actual human body.

\section{Conflict of interest}

The authors declare that they have no conflicts of interest. The authors alone are responsible for the content and writing of the paper.

\section{References}

1. Chen WZ, Xiao Y, Li J. Impact of dose calculation algorithm on radiationtherapy. World J Radiol 2014; 6:874-80.

2. Rana SB. Dose prediction accuracy of anisotropic analytical algorithm and pencil beam convolution algorithm beyond high density heterogeneity interface. South Asian J Cancer 2013; 2:26-30.

3. Sievinen J, Ulmer W, Kaissl W. AAA photon dose calculation model in Eclipse. Palo Alto (CA): Varian Medical Systems, RAD \#7170B; 2005.

4. Gagné IM, Zavgorodni S. Evaluation of the analytical anisotropic algorithm in an extreme water-lung interface phantom using Monte Carlo dose calculations. J Appl Clin Med Phys 2006; 8:33-46.

5. Jabbari K. Review of fast montecarlo codes for dose calculation in radiation therapy treatment planning. J Med Signals Sens 2011; 1:73-86.

6. Rana S. Clinical dosimetric impact of Acuros XB and analytical anisotropic algorithm (AAA) on real lung cancer treatment plans: review. Int J Cancer Ther Oncol 2014; 2:02019.

7. Ojala J. The accuracy of the Acuros XB algorithm in external beam radiotherapy - a comprehensive review. Int J Cancer Ther Oncol 2014; 2:020417.

8. Mallah J, Mihailidis D. SU-E-T-201: Issues encountered in film-based IMRT QA. Med Phys 2011; 38:3532.

9. Andenna C, Benassi M, Caccia B, et al. Comparison of dose distributions in IMRT planning using the gamma function. J Exp Clin Cancer Res 2006; 25:229-34.

10. Nijsten SM, Mijnheer BJ, Dekker AL, et al. Routine individualised patient dosimetry using electronic portal imaging devices. Radiother Oncol 2007; 83:65-75.

11. Huang YC, Yeh CY, Yeh JH, et al. Clinical practice and evaluation of electronic portal imaging device for VMAT qualityassurance. Med Dosim 2013; 38:35-41.

12. Bouchard H, Seuntjens J. Ionization chamber-based reference dosimetry of intensity modulated radiation beams. Med Phys 2004; 31:2454-65.

13. Fraser D, Parker W, Seuntjens J. Characterization of cylindrical ionization chambers for patient spe- cific IMRT QA. J Appl Clin Med Phys 2009; 10:2923.

14. Gurjar OP, Mishra SP, Bhandari V, et al. Radiation dose verification using real tissue phantom in modern radiotherapy techniques. JMed Phys 2014; 39:44-9.

15. ICRU Report 83. Prescribing, recording, and reporting photon-beam intensity-modulated radiation therapy (IMRT). International Commission on Radiation Units and Measurements, Bethesda;2010.

16. Absorbed dose determination in external beam radiotherapy: An international code of practice for dosimetry based on absorbed dose to water. IAEA, Vienna, 2000.

17. Low DA, Moran JM, Dempsey JF, et al. Dosimetry tools and techniques for IMRT. Med Phys 2011; 38:1313-38.

18. Watson PE, Watson ID, Batt RD. Total body water volumes for adult males and females estimated from simple anthropometric measurements. $A m J$ Clin Nutr 1980; 33:27-39.

19. Guyton AC. Textbook of Medical Physiology. $5^{\text {th }}$ edition. Philadelphia: W.B. Saunders; 1976.

20. Guyton AC. Textbook of Medical Physiology. $8^{\text {th }}$ edition. Harcourt College Pub; $8^{\text {th }}$ edition; 1990.

21. Wilson K. Ross and Wilson Anatomy and physiol-

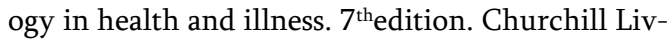
ingstone; 1992.

22. Broerse JJ, Zoetelief J. Dose inhomogeneities for photons and neutrons nearinterfaces. Radiat Prot Dosimetry 2004; 112:509-17.

23. Binger T, Seifert H, Blass G, et al. Dose inhomogeneities on surfaces of different dental implants during irradiation with high-energy photons. Dentomaxillofac Radiol 2008; 37:149-53. 